\title{
Aspirin and clopidogrel resistance using the cone and plate(let) analyser in Indian patients with coronary artery disease
}

\author{
Sudeep Kurien Koshy, Salman Salahuddin, Bijoy Karunakaran, Sajid Yoonus Nalakath, \\ Jayesh Bhaskaran, Padinjare Veloor Haridas, Asishkumar Mandalay, Ali Faizal
}

Department of Cardiology, Malabar Institute of Medical Sciences, Calicut, Kerala, India

\section{Correspondence to}

Dr Ali Faizal, Department of Cardiology, Malabar Institute of Medical Sciences, Calicut, Kerala 673016, India; faizal1ali@yahoo.co.in

Received 11 August 2014 Revised 9 September 2014 Accepted 3 October 2014

\author{
ABSTRACT \\ Background Resistance to antiplatelet drugs is a well- \\ known entity. However, data for aspirin and clopidogrel \\ resistance, and its clinical significance, in Indian patients \\ are meagre.
}

Aims and objectives We sought to determine the prevalence of resistance to aspirin and clopidogrel in Indian patients with stable coronary heart disease (CHD), using the cone and plate(let) analyser (CPA) technology.

Setting and design A single centre prospective study in a cohort of patients with stable CHD on chronic aspirin and clopidogrel therapy attending the cardiology outpatient clinic of a tertiary care hospital in Southern India.

Methods Platelet function was measured using the Impact-R device (DiaMed, Cressier, Switzerland). Resistance to aspirin and clopidogrel was measured in a cohort of 100 patients with stable documented CHD. Relation of antiplatelet resistance to various clinical comorbidities was also assessed.

Results Of the 100 patients, $85 \%$ were men, and $15 \%$ were above 65 years of age. $47 \%$ patients had diabetes, $29 \%$ of patients were hypertensive and $16 \%$ were smokers. Using the CPA, 12 patients (12\%) were found to be resistant to aspirin and 19 patients (19\%) were clopidogrel resistant. In addition, 10 patients (10\%) were resistant to both aspirin and clopidogrel. There was no significant correlation between the presence of antiplatelet resistance and several baseline clinical variables, including age, sex, diabetes,

hypertension and smoking.

Conclusions Resistance to aspirin and clopidogrel and dual antiplatelet resistance are prevalent in Indian patients, comparable with the prevalence worldwide. The CPA is a feasible assay to determine antiplatelet resistance.

\section{INTRODUCTION}

Antiplatelet drug therapy has become standard of care in the management of cardiovascular atherothrombotic disease including acute coronary syndromes and those undergoing percutaneous coronary interventions. ${ }^{12}$ The efficacy of aspirin and clopidogrel in decreasing the risk of adverse cardiac events in patients with coronary heart disease (CHD) has been well established. In spite of this, recurrent atherothrombotic events continue to occur in some patients on chronic oral antiplatelet therapy. Variability in the response to aspirin and clopidogrel therapy has been well described. ${ }^{3-7}$ Non-responsiveness or 'resistance' to the effects of antiplatelet therapy has been studied in recent years by a number of laboratory tests and has been linked to adverse cardiovascular clinical outcomes. ${ }^{8} 9$

CHD in Indian patients has a different epidemiological profile as compared with the Western population, with $\mathrm{CHD}$ occurring at an earlier age, tending to be more aggressive and extensive. Data regarding non-responsiveness to antiplatelet therapy among Indian patients, and its clinical consequences, are scarce and limited. The aim of the present study was to evaluate the prevalence of antiplatelet drug resistance in an Indian population with stable CHD using the cone and plate(let) analyser (CPA) technology.

Various platelet function tests have been devised to assess inborn or acquired abnormal platelet function and also the therapeutic response to antiplatelet agents. Light transmittance aggregometry (LTA) is the most commonly used platelet function test and may be regarded as the gold-standard test for assessing platelet function and also for comparing the efficacy of newer platelet function tests. Other tests include flow cytometry, urinary thromboxane levels, the PFA-100 system and the VerifyNow system, which uses light source to detect platelet aggregation. A relatively newer test on the horizon is the Impact- $\mathrm{R}$ device, which is based on the CPA technology. The device tests platelet adhesion and aggregation in anticoagulated whole blood under arterial shear conditions.

\section{METHODS}

\section{Patients}

We enrolled 100 patients with known CHD attending the outpatient clinic of a tertiary care hospital in South India. Inclusion criteria included those with stable angina with a positive stress test or documented coronary artery disease on a coronary angiogram, documented history of myocardial infarction (MI) (more than 1 month), history of percutaneous coronary intervention and/or coronary artery bypass graft surgery. All patients were on aspirin (enteric coated) $75-150 \mathrm{mg}$ daily and clopidogrel $75 \mathrm{mg}$ daily for a duration of at least 10 days.

Exclusion criteria included acute MI within 30 days of the test, any contraindication to aspirin or clopidogrel therapy, anaemia ( $\mathrm{Hb}<10 \mathrm{~g} \%$ ), renal failure (creatinine $>2.5 \mathrm{mg} / \mathrm{dL}$ ), administration of ticlopidine, dipyridamole or other antiplatelet agents, current non-steroidal anti-inflammatory drug use, administration of heparin or other anticoagulants in the previous $24 \mathrm{~h}$, family or personal history of bleeding disorders, platelet count 
$<150000$ or $>450000$, history of myeloproliferative disorders and major surgical procedures in the last 1 week. All patients gave informed written consent, and the study was approved by the institutional ethics committee.

\section{Measurement of antiplatelet resistance}

The antiplatelet effect of aspirin and clopidogrel was assessed using the Impact-R device (DiaMed, Cressier Morat, Switzerland), which is based on the CPA technology. The device tests platelet adhesion and aggregation in anticoagulated whole blood under arterial shear conditions. The CPA technology is based on applying laminar shear force to whole blood on a polystyrene plate by a rotating cone, leading to platelet surface adhesion and aggregation. Samples of whole blood $(130 \mu \mathrm{L})$ anticoagulated with sodium citrate were placed on polystyrene wells and subjected to flow at $1800 \mathrm{~s}^{-1}$ for 2 min using a special conical disc. After the surface is washed with phosphatebuffered saline and stained, samples were analysed by an image analyser. The results are expressed as a percentage of the well surface covered (SC) by platelets and as average size of the adherent aggregate particles. Blood samples were pretreated with agonists arachidonic acid (AA) and ADP, to assess specifically antiplatelet response to aspirin and clopidogrel, respectively.

\section{Aspirin assay}

For assessing resistance to aspirin, the blood sample was pretreated with AA $(0.32 \mathrm{mM})$ under gentle mixing $(10 \mathrm{rpm})$ for $1 \mathrm{~min}$ and then subjected to the regular Impact- $\mathrm{R}$ test. A result of a low SC suggests that the platelets do not respond to aspirin. A percentage SC of $\geq 2.5 \%$ was considered as cut-off value indicating adequate response to aspirin, whereas a value $<2.5 \%$ indicates resistance to aspirin. ${ }^{10} 11$

\section{Clopidogrel assay}

For assessing response to clopidogrel, the blood sample was pretreated with ADP $(1.38 \mu \mathrm{M})$ under gentle mixing $(10 \mathrm{rpm})$ for $1 \mathrm{~min}$ and then subjected to the regular Impact test. A result of a low SC suggests that the platelets do not respond to clopidogrel. A percentage SC of $\geq 2.8 \%$ was considered as cut-off value indicating adequate response to clopidogrel, whereas a value $<2.8 \%$ indicated resistance. ${ }^{10} 11$

\section{Statistical analysis}

To ensure sample adequacy in the survey, we conducted power analysis to find the sample size required for a power that is $>90 \%$. Considering that previous studies have estimated an antiplatelet resistance of 5\%-45\% in patients taking aspirin and $4 \%-30 \%$ in patients taking clopidogrel, ${ }^{12-16}$ we took an estimated prevalence of $30 \%$ to calculate the sample size. Taking this into account the sample size obtained statistically was 98 .

Categorical variables are expressed as frequencies and percentages. Differences between groups were assessed with the Fisher exact test for categorical variables. Unpaired t tests were used for comparison of normally distributed continuous variables between the 2 groups. A p value $<0.05$ was considered statistically significant. Statistical analysis was performed with SPSS V.17.0 software (SPSS, Chicago, USA).

\section{RESULTS}

This study analysed the prevalence of resistance to aspirin and clopidogrel in 100 patients with CHD in an urban tertiary level care hospital in North Kerala in India. Majority of the patients $(85 \%)$ were men and $15 \%$ of the patient population were above 65 years. The prevalence of diabetes in the total population was
Table 1 Baseline characteristics of all patients in the study $(n=100)$

Total $(n=100)$

\begin{tabular}{lc} 
Advanced age (>65 years) & $15(15 \%)$ \\
Male sex & $85(85 \%)$ \\
Diabetes & $47(47 \%)$ \\
Hypertension & $29(29 \%)$ \\
Smoking & $16(16 \%)$ \\
Family history of CHD & $13(13 \%)$ \\
Clinical characteristics & \\
$\quad$ Chronic stable angina & $21(21 \%)$ \\
History of recent NSTEACS & $41(41 \%)$ \\
History of recent STEMI & $27(27 \%)$ \\
Heart failure & $11(11 \%)$ \\
Drug intake & \\
$\beta$-Blocker & $69(69 \%)$ \\
Calcium channel blocker & $20(20 \%)$ \\
ACE inhibitor & $71(71 \%)$ \\
Aldosterone antagonist & $20(20 \%)$ \\
Statin & $100(100 \%)$ \\
\hline
\end{tabular}

CHD, coronary heart disease; NSTEACS, non-ST elevation acute coronary syndrome; STEMI, ST elevation myocardial infarction.

$47 \%$, and $29 \%$ were hypertensive. Sixteen per cent $(16 \%)$ of the total population were smokers (table 1).

Using the Impact-R CPA, the percentage of patients with coronary artery disease having aspirin resistance was $12 \%$, and clopidogrel resistance was found in 19\% patients. Furthermore, $10 \%$ of patients showed resistance to the antiplatelet effect of both aspirin and clopidogrel (figure 1).

We also assessed the correlation of antiplatelet resistance with clinical variables, namely age, sex, diabetes, hypertension, smoking status, family history of coronary artery disease and concurrent medications. None of the clinical variables showed statistically significant correlation with antiplatelet resistance (table 2).

\section{DISCUSSION}

Significant heterogeneity exists among global studies reporting prevalence of aspirin and clopidogrel resistance. Lack of a goldstandard investigation and variability in the methods of assessing antiplatelet resistance are largely responsible for this heterogeneity. For aspirin, difference in prevalence in various studies may be due to variability in doses. In the case of clopidogrel, this difference can be attributed to variability in definitions, populations studied, laboratory methods and agonist doses within the same laboratory assay. ${ }^{7}$

Reported prevalence of aspirin non-responsiveness varies widely from $0 \%$ to $57 \%$. In a systematic review by Hovens et al, ${ }^{17}$ the mean prevalence of aspirin resistance was $24 \%$. In this analysis, studies using light aggregometry with AA as an agonist had a pooled prevalence of $6 \%$ (95\% CI $0 \%$ to $12 \%)$, whereas in studies using point-of-care assays, the prevalence was higher at 26\% (95\% CI 21\% to 31\%). Similarly, studies evaluating prevalence of clopidogrel resistance have also showed considerable heterogeneity. In a study of 150 patients undergoing percutaneous coronary intervention, clopidogrel resistance using LTA was $24 \%{ }^{18}$ Buonamici et $a l^{19}$ studied 804 patients using ADP-induced platelet aggregation and reported a prevalence of $13 \%$.

Studies from the Indian subcontinent and South East Asia, evaluating antiplatelet resistance, are scarce. Sadiq et $a l^{20}$ showed 


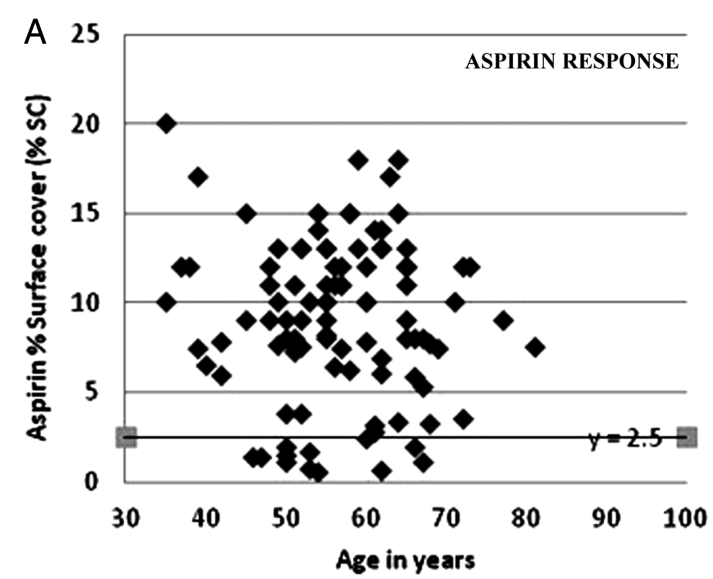

Figure 1 Distribution of patients with aspirin and clopidogrel resistance.

that nearly $42 \%$ of patients with CHD have inadequate response to aspirin. Akhtar et $a l^{21}$ studied aspirin resistance in 250 patients with stable CHD and showed that $12 \%$ of patients were resistant to aspirin. In the study conducted by Guha et al, ${ }^{22}$ in patients with acute coronary syndrome, $15.27 \%$ showed resistance to aspirin, $19.44 \%$ were resistant to clopidogrel and $12.5 \%$ were resistant to both. In another study involving patients with recurrent acute coronary syndromes, aspirin, clopidogrel and dual antiplatelet resistance were encountered, respectively, in $35 \%$, $72.5 \%$ and $32.5 \%$ patients with recurrent acute coronary syndrome undergoing conservative management. ${ }^{23}$

In our study population, aspirin resistance was found in $12 \%$ of patients, clopidogrel resistance in $19 \%$ of patients and $10 \%$ patients were found to be resistant to both antiplatelet drugs. The values obtained in our study are comparable with previous existing data. Only patients with stable CHD were included in our study. Whether differences exist between antiplatelet responsiveness in stable $\mathrm{CHD}$ and acute coronary syndromes is

Table 2 Baseline clinical characteristics of patient groups according to antiplatelet resistance

\begin{tabular}{lccc}
\hline & $\begin{array}{l}\text { Aspirin } \\
\text { resistant } \\
(\mathbf{n}=12)\end{array}$ & $\begin{array}{l}\text { Clopidogrel } \\
\text { resistant } \\
(\mathbf{n}=19)\end{array}$ & $\begin{array}{l}\text { Dual } \\
\text { resistant } \\
(\mathbf{n}=10)\end{array}$ \\
\hline Advanced age (>65 years) & $2(16.7 \%)$ & $5(26.3 \%)$ & $2(20.0 \%)$ \\
Male sex & $11(91.7 \%)$ & $16(44.2 \%)$ & $9(90 \%)$ \\
Diabetes & $4(33.3 \%)$ & $8(42.1 \%)$ & $3(30 \%)$ \\
Hypertension & $5(41.7 \%)$ & $10(52.6 \%)$ & $4(40 \%)$ \\
Smoking & $1(8.3 \%)$ & $2(10.5 \%)$ & $1(10 \%)$ \\
$\begin{array}{l}\text { Family history of CHD } \\
\text { Clinical characteristics }\end{array}$ & $2(16.7 \%)$ & $1(5.3 \%)$ & $1(10 \%)$ \\
$\quad$ Chronic stable angina & $4(33.3 \%)$ & $5(26.3 \%)$ & $4(40 \%)$ \\
$\quad$ History of recent & $3(25 \%)$ & $5(26.3 \%)$ & $2(20 \%)$ \\
NSTEACS & $4(33.3 \%)$ & $8(42.1 \%)$ & $4(40 \%)$ \\
$\quad$ History of recent STEMI & $1(8.3 \%)$ & $1(5.3 \%)$ & $0(0 \%)$ \\
$\quad$ Heart failure & $8(66.7 \%)$ & $13(68.4 \%)$ & $8(80 \%)$ \\
Drug intake & $4(33.3 \%)$ & $5(26.3 \%)$ & $3(30 \%)$ \\
$\quad \begin{array}{l}\text { B-Blocker } \\
\text { Calcium channel blocker }\end{array}$ & $10(83.3 \%)$ & $15(78.9 \%)$ & $7(70 \%)$ \\
$\quad \begin{array}{l}\text { ACE inhibitor } \\
\text { Aldosterone antagonist }\end{array}$ & $1(8.3 \%)$ & $5(26.3 \%)$ & $1(10 \%)$ \\
\hline
\end{tabular}

Data are $n(\%)$ unless noted otherwise. There were no significant $(p<0.05)$ differences between these groups.

CHD, coronary heart disease; NSTEACS, non-ST elevation acute coronary syndrome; STEMI, ST elevation myocardial infarction.

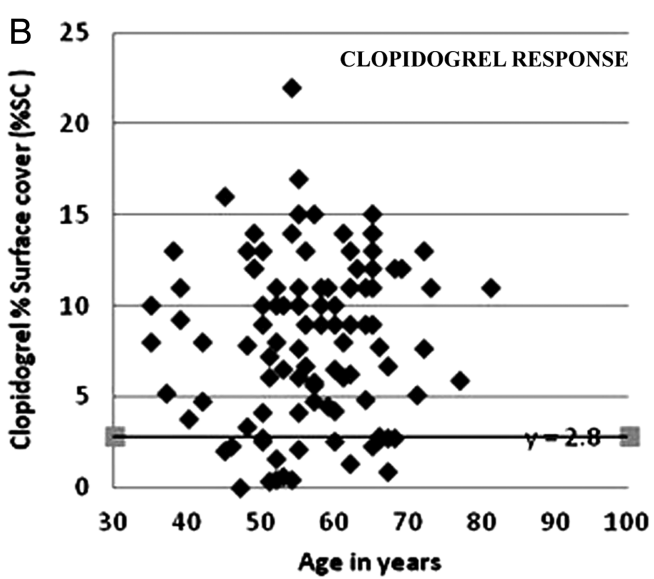

unclear. Differences also exist in platelet reactivity in different clinical conditions. Obesity, diabetes mellitus, hypercholesterolaemia, smoking and heart failure have been shown to be associated with increased degree of platelet reactivity. ${ }^{7}$ We could, however, find no statistically significant correlation between antiplatelet resistance and various clinical variables in our study.

Several assays exist to measure antiplatelet efficacy and resistance. These include flow cytometry, LTA, urinary thromboxane levels and several point-of-care tests like the VerifyNow assay. In an antiplatelet resistance study by Thomson et $a l^{24}$ using urinary 11-dehydrothromboxane B2 levels as a surrogate marker for antiplatelet efficacy, aspirin resistance was found to be $38.1 \%$. Between the major platelet function tests there has been poor correlation in determining the prevalence of aspirin resistance as illustrated by Lordkipanidzé $e t a l^{25}$ in their study. The prevalence of aspirin resistance varied according to the assay used: $10.3 \%-51.7 \%$ for LTA using ADP as the agonist, $18.0 \%$ for whole blood aggregometry, $59.5 \%$ for PFA-100, $6.7 \%$ for VerifyNow Aspirin and $22.9 \%$ by measuring urinary 11-dehydrothromboxane $\mathrm{B}(2)$ concentrations. The rate of clopidogrel resistance is also dependent on the assay used. In a study of 70 patients receiving $150 \mathrm{mg}$ clopidogrel after coronary stenting, the clopidogrel resistance was $13 \%$ with LTA using ADP as agonist, 39\% with vasodilator-stimulated phosphoprotein assay and 33\% with the VerifyNow P2Y12 assay. ${ }^{26}$ Moreover, it has also been shown that aspirin resistance may not be a stable feature over time, may be transient and thus assessment at a single point of time may not hold true subsequently. ${ }^{27} 28$

Compared with the various modalities of detecting antiplatelet resistance, the CPA used in our study assesses platelet function in physiological circumstances, is not time-consuming and can be easily done by trained lab personnel. Several studies have used the CPA to assess antiplatelet efficacy and have shown good correlation with currently accepted standards. ${ }^{29} 30$

Antiplatelet therapy is the cornerstone in the primary and secondary prevention of acute and chronic coronary and cerebrovascular syndromes. Knowledge of the efficacy of these drugs is therefore of paramount importance, especially since there are alternatives like the new generation P2Y12 antagonists prasugrel and ticagrelor, which may be given to patients to overcome antiplatelet resistance. Our study shows that south Indian population also has a similar prevalence of individual and dual antiplatelet resistance. Further larger studies are needed to compare the efficacy of individual antiplatelet agents and to assess the effect of variables like the effect of diabetes, dyslipidaemia and smoking on antiplatelet resistance. The CPA may be a simple method to assess antiplatelet resistance, especially in 
patients with recurrent thrombotic events, stent thrombosis and other high risk population like diabetics.

\section{STUDY LIMITATIONS}

This study was done in a hospital setting on patients with stable CHD receiving treatment in the cardiology department. This selection bias limits the extent to which our results can be projected to the general population. Comparison of antiplatelet resistance using currently accepted assays like LTA and point-of-care tests like VerifyNow, with the Impact-R test used in our study, was not performed. Also, clinical outcomes of the patients with biochemical antiplatelet resistance were not studied in our study population.

\section{CONCLUSIONS}

Aspirin, clopidogrel and dual antiplatelet resistance is common in South India and is comparable with the values obtained globally. The prevalence of aspirin, clopidogrel and dual antiplatelet resistance in the study population was $12 \%, 19 \%$ and $10 \%$, respectively. The CPA is a simple and feasible method to determine antiplatelet resistance. Further studies may be required to compare efficacy of this assay with currently accepted standards.

Contributors $A F, A M$ and SKK were involved in conception and design of the study. SKK and SS were involved in acquisition, analysis and interpretation of data and drafting of the manuscript. AF, BK, JB, SYN and PVH were involved in critical review of the manuscript.

Competing interests None.

Patient consent Obtained.

Ethics approval Institutional Ethics Committee- Malabar Institute of Medical Sciences, Calicut, Kerala, India.

Provenance and peer review Not commissioned; externally peer reviewed.

\section{REFERENCES}

1 Chen ZM, Jiang LX, Chen YP, et al. Addition of clopidogrel to aspirin in 45,852 patients with acute myocardial infarction: randomised placebo-controlled trial. Lancet 2005;366:1607-21.

2 Steinhubl SR, Berger PB, Mann JT III, et al. Early and sustained dual oral antiplatelet therapy following percutaneous coronary intervention: a randomized controlled trial. JAMA 2002;288:2411-20.

3 Buchanan MR, Brister SJ. Individual variation in the effects of ASA on platelet function: implications for the use of ASA clinically. Can J Cardiol 1995;11:221-7.

4 Eikelboom JW, Hirsh J, Weitz Jl, et al. Aspirin-resistant thromboxane biosynthesis and the risk of myocardial infarction, stroke, or cardiovascular death in patients at high risk for cardiovascular events. Circulation 2002;105:1650-5.

5 Serebruany VL, Steinhubl SR, Berger PB, et al. Variability in platelet responsiveness to clopidogrel among 544 individuals. J Am Coll Cardiol 2005;45:246-51.

6 Dupont AG, Gabriel DA, Cohen MG. Antiplatelet therapies and the role of antiplatelet resistance in acute coronary syndrome. Thromb Res 2009;124:6-13.

7 Kuliczkowski W, Witkowski A, Polonski L, et al. Inter-individual variability in the response to oral antiplatelet drugs: a position paper of the Working Group on antiplatelet drugs resistance appointed by the Section of Cardiovascular Interventions of the Polish Cardiac Society, endorsed by the Working Group on Thrombosis of the European Society of Cardiology. Eur Heart J 2009;30:426-35.
8 Gurbel PA, Bliden KP, Guyer K, et al. Platelet reactivity in patients and recurrent events post-stenting: results of the PREPARE POST-STENTING Study. J Am Coll Cardiol 2005;46:1820-6.

9 Geisler T, Langer $\mathrm{H}$, Wydymus $\mathrm{M}$, et al. Low response to clopidogrel is associated with cardiovascular outcome after coronary stent implantation. Eur Heart J 2006;27:2420-5.

10 Spectre G, Brill A, Gural A, et al. A new point-of-care method for monitoring antiplatelet therapy: application of the cone and plate(let) analyzer. Platelets 2005; 16:293-9.

11 Savion N, Varon D. Impact- The Cone and Plate(let) Analyzer: testing platelet function and antiplatelet drug response. Pathophysiol Hemost Thromb 2006:35:83-8.

12 Gurbel PA, Bliden KP, Hiatt BL, et al. Clopidogrel for coronary stenting: response variability, drug resistance, and the effect of pretreatment platelet reactivity. Circulation 2003;107:2908-13.

13 Gum PA, Kottke-Marchant K, Welsh PA, et al. A prospective, blinded determination of the natural history of aspirin resistance among stable patients with cardiovascular disease. J Am Coll Cardiol 2003;41:961-5.

14 Gum PA, Kottke-Marchant K, Poggio ED, et al. Profile and prevalence of aspirin resistance in patients with cardiovascular disease. Am J Cardiol 2001;88:230-5.

15 Grotemeyer KH. Effects of acetylsalicylic acid in stroke patients. Evidence of nonresponders in a subpopulation of treated patients. Thromb Res 1991;63:587-93.

16 Muller I, Besta F, Schulz C, et al. Prevalence of clopidogrel nonresponders among patients with stable angina pectoris scheduled for elective coronary stent placement. Thromb Haemost 2003;89:783-7.

17 Hovens MM, Snoep JD, Eikenboom JC, et al. Prevalence of persistent platelet reactivity despite use of aspirin: a systematic review. Am Heart J 2007;153:175-81.

18 Lev El, Patel RT, Maresh KJ, et al. Aspirin and clopidogrel drug response in patients undergoing percutaneous coronary intervention: the role of dual drug resistance. $J$ Am Coll Cardiol 2006;47:27-33.

19 Buonamici P, Marcucci R, Migliorini A, et al. Impact of platelet reactivity after clopidogrel administration on drug-eluting stent thrombosis. J Am Coll Cardiol 2007:49:2312-7.

20 Sadiq PA, Puri A, Dixit $M$, et al. Profile and prevalence of aspirin resistance in Indian patients with coronary artery disease. Indian Heart J 2005;57:658-61.

21 Akhtar N, Junaid A, Khalid A, et al. Frequency of aspirin resistance in patients with coronary artery disease in Pakistan. Pak J Pharm Sci 2009;22:230-3.

22 Guha S, Sardar P, Guha P, et al. Dual antiplatelet drug resistance in patients with acute coronary syndrome. Indian Heart J 2009;61:68-73.

23 Guha S, Mookherkee S, Guha P, et al. Antiplatelet drug resistance in patients with recurrent acute coronary syndrome undergoing conservative management. Indian Heart J 2009;61:348-52.

24 Thomson VS, John B, George $P$, et al. Aspirin resistance in Indian patients with coronary artery disease and cardiovascular events. J Postgrad Med 2009;55:252-6.

25 Lordkipanidzé M, Pharand C, Schampaert E, et al. A comparison of six major platelet function tests to determine the prevalence of aspirin resistance in patients with stable coronary artery disease. Eur Heart J 2007;28:1702-8.

26 Cuisset T, Frere C, Poyet R, et al. Clopidogrel response: head-to-head comparison of different platelet assays to identify clopidogrel non-responder patients after coronary stenting. Arch Cardiovasc Dis 2010;103:39-45.

27 Santilli F, Rocca B, De Cristofaro R, et al. Platelet cyclooxygenase inhibition by low-dose aspirin is not reflected consistently by platelet function assays: implications for aspirin "resistance". J Am Coll Cardiol 2009;53:667-77.

28 Lev El. Aspirin resistance transient laboratory finding or important clinical entity? J Am Coll Cardiol 2009;53:678-80.

29 Shenkman B, Einav Y, Salomon 0, et al. Testing agonist-induced platelet aggregation by the Impact- $R$ [Cone and plate(let) analyzer (CPA)]. Platelets 2008;19:440-6.

30 Morrison A, Hornsey VS, Prowse CV, et al. Use of the DiaMed Impact R to test platelet function in stored platelet concentrates. Vox Sanguinis 2007;93:166-72. 